\title{
Integrating Electrochemical and Colorimetric Sensors with a Webcam Readout for Multiple Gas Detection
}

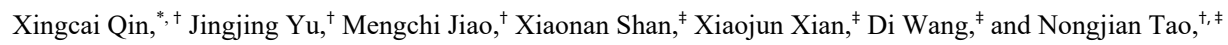 \\ ${ }^{\dagger}$ State Key Laboratory of Analytical Chemistry for Life Science, School of Chemistry and Chemical Engineering, Nanjing \\ University, Nanjing, Jiangsu 210023, China \\ ${ }^{\ddagger}$ Biosensor and Bioelectronics Center, the Biodesign Institute, Arizona State University, Tempe, Arizona 85287, United States. \\ Correspondence email: xingcai.qin@nju.edu.cn
}

Table of contents

SI 1. Materials and instruments

SI 2. Structure of the multiple sensor chamber.

SI 3. Schematic of the sensing platform

SI 4. Equation derivation for quantitative analysis of $\mathrm{BPE} \mathrm{O}_{2}$ sensor

SI 5. Thickness of Au layer sputtered on the Ag surface

SI 6. Video of Ag layer electrodissolution

SI 7. Edge recognition algorism

SI 8. Reference

\section{SI 1. Materials and instruments}

The high purity gas of $\mathrm{N}_{2}, \mathrm{CO}_{2}, \mathrm{O}_{2}, 15.9 \% \mathrm{O}_{2}, 20.9 \% \mathrm{O}_{2}, 30 \mathrm{ppmV} \mathrm{NO}_{2}, 50 \mathrm{ppmV} \mathrm{SO}_{2}$ and 50 ppmV HCHO (balanced with $\mathrm{N}_{2}$ ) are from gas cylinder supplied by Tianze gas Co, Ltd. Gas with other concentrations are diluted in sampling bag (outer layer of Tedlar and inner layer of PVDF from Dalian Hade Co, Ltd. ) with proportional target gas and balance gas $\left(\mathrm{N}_{2}\right)$, whose volume quantitated by syringe $(10 \mathrm{~mL}, 20 \mathrm{~mL}, 50 \mathrm{~mL}$ ) or electric flowmeter (MF5700-N-10 from Siargo Ltd.). In addition, their concentrations are further confirmed by corresponding 
commercial detectors. $\mathrm{O}_{2}$ detector, $\mathrm{CO}_{2}$ detector, $\mathrm{SO}_{2}$ detector are PGM-1600 from RAE, Telaire T7001, and SGA-600-SO2 from Sigoan respectively.

Gas channel is composed of flexible Tygon tube (inner layer of PTFE) and luer connectors, both of which are from Cole-Parmer. Used to control and balance humidity, nafion tube with diameter of $2 \mathrm{~mm}$ is from Perma Pure LLC. Mini pump for gas delivery is KVP04 from Kamoer. Electronic valve is 0520D from Fospump. Sensor chamber is made from transparent PMMA and PTFE by CNC machine (Xendoll C00003A) and laser cutter (optima-5070, Nanjing Chaohan Co, Ltd). Webcam is Logitech C525. DC Power supply is DP832 from RIGOL. CHI 760E electrochemical workstation was used to supply driving voltage on the steel driving electrode and reference Pt electrode. Humidity monitor is RH390 from EXTECH. Ion sputter coater for Au coating is SBC-12 from KYKY. Pure water $(18.2 \mathrm{M} \Omega \bullet \mathrm{cm})$ is from MilliQ pure water system.

The BPE with Ag layer is exploited from HP 16x CD and sputtered with Au layer. Ion sputter coater was used to sputter Au on the top and bottom with thickness of $\sim 1.6 \mathrm{~nm}$ ( $20 \mathrm{~s}$ sputtering) and $\sim 26 \mathrm{~nm}$ (sputtering time of $320 \mathrm{~s}$ ) respectively. After coating, the conductance of bottom Au was $\sim 2 \Omega$, which could hold potential gradients along the BPE.

The EC aqueous solution is composed of $\mathrm{NaH}_{2} \mathrm{PO}_{4}(10 \% \mathrm{wt}), \mathrm{KCl}(2 \% \mathrm{wt})$, anti-foam SE-15 $(1 \% \mathrm{~V})$, and all the chemicals are all from sigma-aldrich. Anti-foam 15 help decrease bubbling at driving electrode, which would further decrease the electrolytic $\mathrm{O}_{2}$ influence on target oxygen and bubbling induced possible movement and intensity change of BPE electrode.

The recipe of the $\mathrm{NO}_{2}$ sensor is a water solution composing of $4 \mathrm{ml}$ water, $0.4 \mathrm{~g} \mathrm{~N}$,N-dimethylp-phenylenediamine sulfate, and $0.5 \mathrm{ml}$ glycerol. Silicon paper, which is used as substrate to load sensing chemicals, is TLC plates silica gel matrix with dimension of $20 \mathrm{~cm}(\mathrm{~L}) \times 20 \mathrm{~cm}$ (W) $\times 250 \mu \mathrm{m}$ (thickness) from Sigmal-Aldrich (lot\# 3110). The substrate of Silica paper was cut into piece of $10 \mathrm{~mm} \times 20 \mathrm{~mm}$, immersed in the sensor solution, shaken with orbital shaker (TOS-200 from TiTan Scientific Lab) at $100 \mathrm{rpm}$ for $30 \mathrm{~min}$, and dried in vacuum chamber overnight. The sensor chip was cut into $5 \mathrm{~mm} \times 5 \mathrm{~mm}$ for subsequent sensor test.

SI 2. Structure of the multiple sensor chamber. 

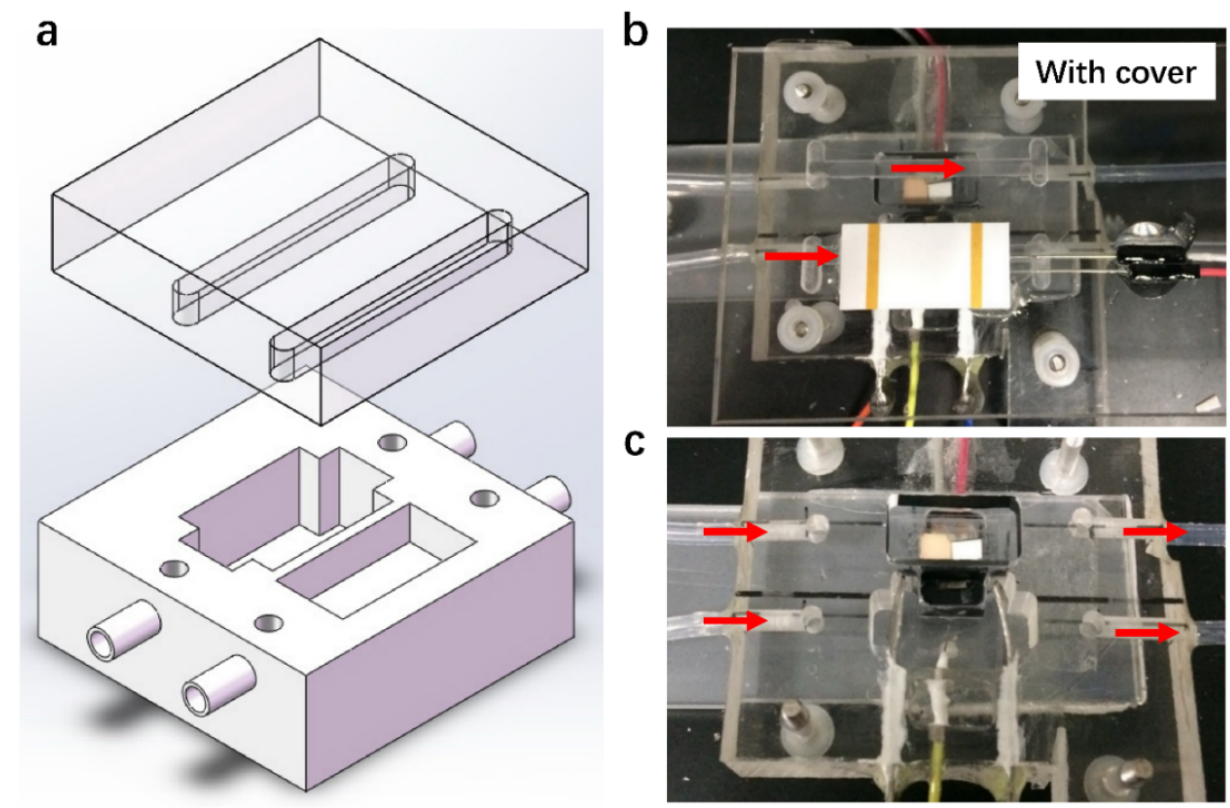

Figure S1. (a) 3D model of the multiple sensors chamber including separated colorimetric sensor cell and BPE sensor cell together with top transparent cover. The holes in the chamber and grooves in the cover composed parallel gas channels. Images of the sensor chamber with (b) and without top cover (c). Gas channels are red arrowed.

\section{SI 3. Schematic of the sensing platform}

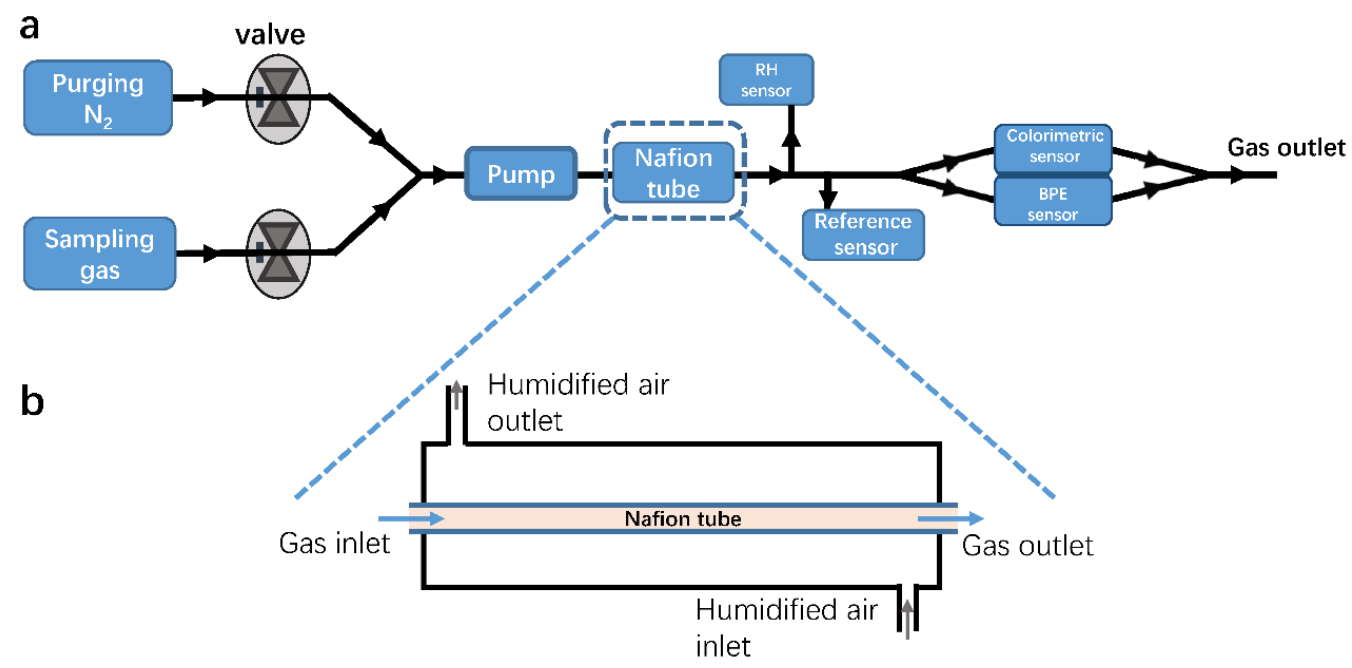

Figure S2. (a) Schematic of the sensing platform and its gas delivering system. Two 2-way electronic valves were used to switch purging and sampling gas alternatively, which can supply more stable flowrate than one 3-way valve to avoid switching induced pulse flowrate change. Pumped from sampling bags, gas passed through nafion tubing, sensor chamber (with parallel sensor cells) in sequence. Reference sensors were used to confirm gas concentration and humidity in need. (b) The humidity control 
system based on nafion tubes with an outer PTFE tube and inner nafion tube. Air with set humidity passed through the outer PTFE tube in opposite direction to gas through inner nafion tubes, which is sampling gas or purging $\mathrm{N}_{2}$ from sampling bags. Nafion tube, allowing water molecule permeation, would balance humidity of the inner gas with outer humidified gas and make the sampling gas/purging $\mathrm{N}_{2}$ have the same humidity with outer humidified air. The length of nafion tube used here is $1.5 \mathrm{~m}$ long with diameter of $2 \mathrm{~mm}$, which is sufficient for humidity balance at the flowrate of 1 LPM.

\section{SI 4. Equation derivation for quantitative analysis of $B P E \mathrm{O}_{2}$ sensor}

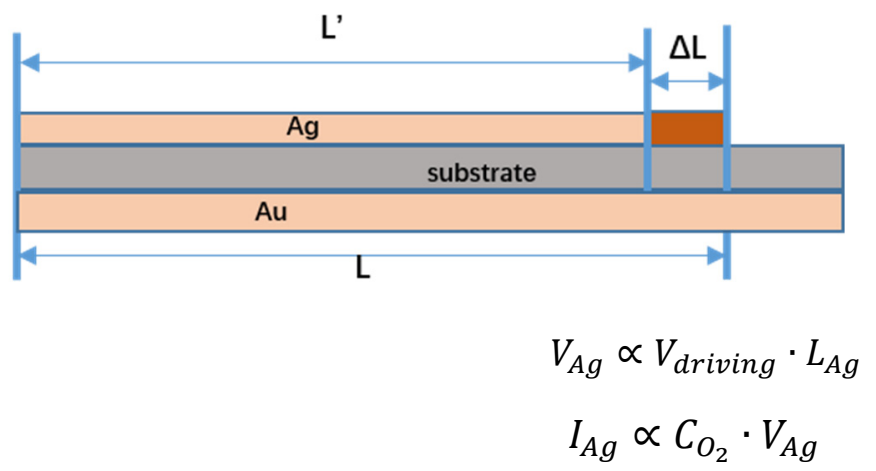

For short dissolving length of Ag layer on the BPE labelled as $\Delta L_{A g}$,

$$
\begin{gathered}
Q=t \cdot I_{A g}=1 \cdot n_{A g_{\text {dissolving }}}=\frac{m_{A g}}{M_{A g}}=\frac{\rho \cdot V}{M_{A g}}=\frac{\rho * \Delta L_{A g * W * \sigma}}{M_{A g}} \\
\frac{\Delta L_{A g}}{t} \cdot w \propto C_{O_{2}} \cdot V_{\text {driving }} \cdot L_{A g}
\end{gathered}
$$

Where, $V_{d r i v i n g}, V_{A g}$ and $I_{A g}$ are the driving voltage between driving electrode and reference electrode, potential drop between two ends of the present Ag layer, and EC current cross the present Ag layer respectively. $n_{A g}$ dissolving, $\Delta L_{A g}, m_{A g}, W$ and $\sigma$ are amount of substance (mol), the length, the mass, the width, and the thickness of dissolved Ag layer. While $L_{A g}$ represents the length of present $\mathrm{Ag}$ layer before the dissolving.

\section{SI 5. Thickness of Au layer sputtered on the Ag surface}

The Au layer is sputtered by SBC-12 ion sputter coater, which is from KYKY technology Co, Ltd. The sputtering time is $20 \mathrm{~s}$ and $320 \mathrm{~s}$ for top and bottom Au layer respectively with sputtering current of $7 \mathrm{~mA}$ and vacuum of $6 \mathrm{~Pa}$. The thickness is calculated by empirical equation:

$$
\mathrm{d}(\AA)=\mathrm{K}^{*} \mathrm{I}^{*} \mathrm{~V} * \mathrm{~T}
$$

In this experiment, $\mathrm{K}=0.07, \mathrm{I}=7 \mathrm{~mA}$, and $\mathrm{V}=1.68 \mathrm{KV}$. 
Therefore, for top Au layer (sputtering time of $20 \mathrm{~s}$ )

$$
\mathrm{d}_{20}=0.07 * 7 * 1.68 * 20=16 \AA
$$

when sputtering time is $30 \mathrm{~s}$ :

$$
\mathrm{d}_{30}=0.07 * 7 * 1.68 * 30=25 \AA
$$

for supporting bottom Au layer (sputtering time of 320 s)

$$
\mathrm{d}_{320}=0.07 * 7 * 1.68 * 320=263 \AA
$$

It should be noted that thicker top Au layer than 2.5 would block the $\mathrm{Ag}$ dissolution and further block immediate detection of Ag layer. OM images also indicated that $20 \mathrm{~s}$ sputtering might not produce a continuous Au layer.

\section{SI6. Video of Ag electrode electrodissolution.}

Videos of Ag electrode electrodissolution were attached. The original video showed 2 typical detection cycles with different $\mathrm{O}_{2}$ concentrations at frame rate of 5 frames per second (fps). The $\mathrm{Ag}$ dissolution happened at $96 \mathrm{~s}$ and $270 \mathrm{~s}$. To make it clear, the video accelerated by 20 times ( $100 \mathrm{fps}$ ) was also attached.

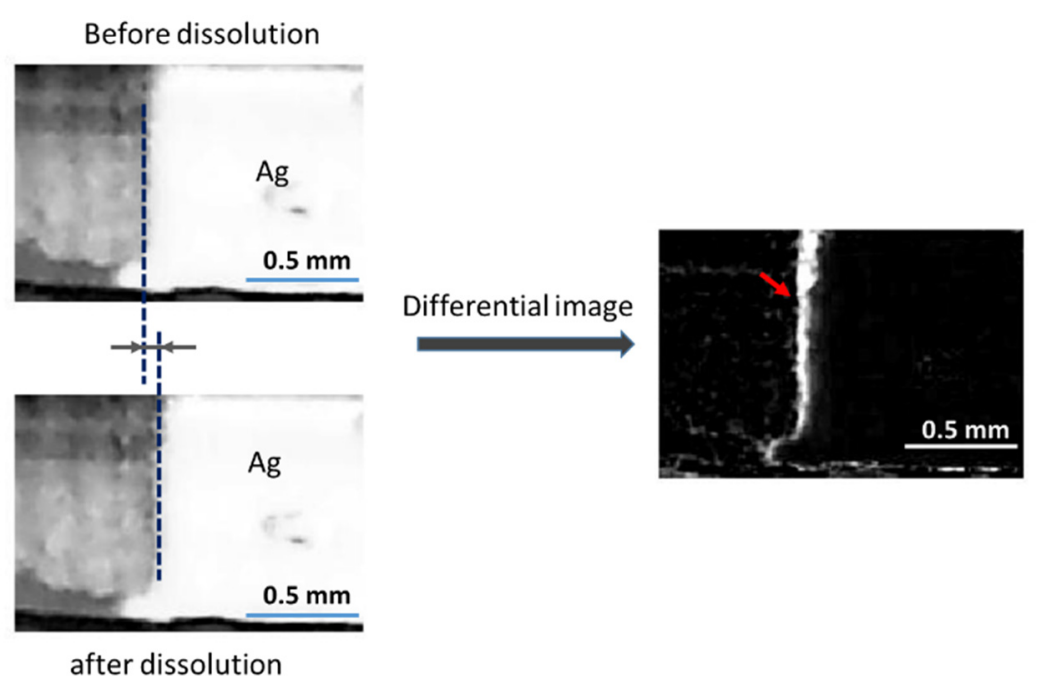

Figure S3. The differential image between images before and after Ag dissolution extracted from the attached video. The black dash lines showed the edges of the Ag layer. The decreased length was also labelled. The red arrow indicated the position of the dissolved Ag, which is shown as light region.

\section{SI 7. Edge recognition algorism}



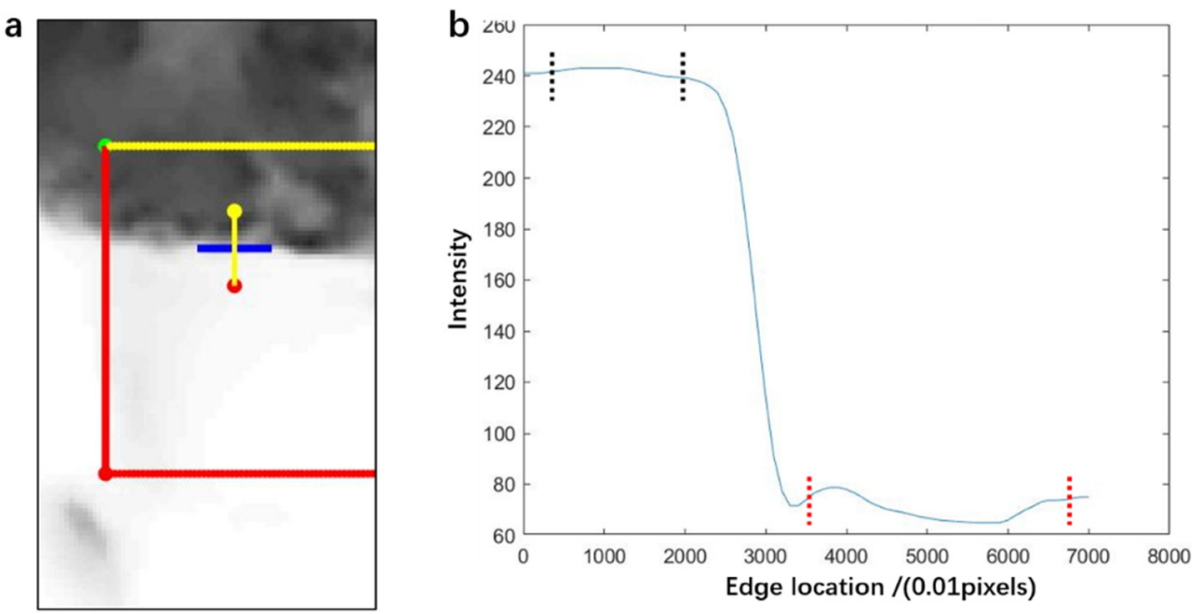

Figure S4. Edge recognition algorism. Generally, a line crossing light area (Ag layer) and dark area (substrate revealed after Ag dissolution), which is vertical to border between light and dark, was used to find the edge according to intensity of each pixel on the line. The location (sequence) of the pixels whose intensity is the mid value between the light and dark area is set as the edge location. (a) Image of BPE, Ag layer (light area) and border (blue line). Pixels on the horizontal yellow line and red line were the starting points and endpoints. Vertical red line, which is vertical to the Ag border (blue line), was the direction from starting points to endpoints. The curves from starting points to corresponding endpoints along the direction line were used to find the Ag edge as shown in (b). (b) A typical curve used to find edge, which is from start points (a pixel on the yellow horizontal line) to endpoints (corresponding pixels on the horizontal red line) along the direction of vertical red line. Each data point was the pixel sequence (x-axis) and its intensity (y-axis), which is further linearly interpolated with equal 100 data points for each adjacent pixels. The mid value of intensity between light area (mean value between black dash lines) and dark area (mean value between red dash lines) was set as the anchor value to find corresponding pixel sequence as the edge location, which is also used to find edge locations of other curves. Meaning the edge values of each curve was the edge of this frame image. And edge location of time sequential images gave the response curve shown in figure $3 \mathrm{a}$.

\section{Reference:}

1 Fosdick, S. E., Knust, K. N., Scida, K. \& Crooks, R. M. Bipolar Electrochemistry. Angew Chem Int Edit 52, 10438-10456, doi:10.1002/anie.201300947 (2013). 
2 Duval, J. F. L., Huijs, G. K., Threels, W. F., Lyklema, J. \& van Leeuwen, H. P. Faradaic depolarization in the electrokinetics of the metal-electrolyte solution interface. J Colloid Interf Sci 260, 95-106, doi:10.1016/S0021-9797(02)00134-0 (2003). 